Check for updates

Cite this: J. Mater. Chem. A, 2021, 9, 4898

Received 26th September 2020 Accepted 18th January 2021

DOI: 10.1039/d0ta09462e

rsc.li/materials-a

\section{Unravelling thermal stress due to thermal expansion mismatch in metal-organic frameworks for methane storage $\uparrow$}

\author{
Jelle Wieme (D) and Veronique Van Speybroeck (D)*
}

\section{Introduction}

Temperature fluctuations inducing thermal stress impact the operation of many technological applications. This particularly applies to systems composed of various material constituents in close contact with each other. For example, internal stress arises when the volume response upon varying temperature differs between two components. This so-called thermal expansion mismatch may lead to system failures due to cracks or plastic deformation, ${ }^{1,2}$ but was recently also exploited to stabilize otherwise inaccessible material phases. ${ }^{3}$

In this study, we focus on metal-organic frameworks (MOFs). This promising class of porous, crystalline materials has mainly been investigated for adsorption-based technologies, ${ }^{4-6}$ and distinguishes itself from other candidate materials by its chemical and physical versatility. ${ }^{4}$ These applications where adsorption and desorption processes lie at the basis of their operation might especially be prone to thermal stress as the exchange of guest molecules with the porous framework could lead to large temperature changes without a proper heat management strategy. ${ }^{7-9}$ Moreover, the temperature is sometimes also used as control parameter for adsorption or

Center for Molecular Modeling, Ghent University, Tech Lane Ghent Science Park Campus A, Technologiepark 46, 9052 Zwijnaarde, Belgium. E-mail: veronique. vanspeybroeck@ugent.be

$\dagger$ Electronic supplementary information (ESI) available. See DOI: 10.1039/d0ta09462e desorption of guest molecules in some setups, i.e. temperature swing adsorption (TSA). ${ }^{10}$

Up to now, there has been limited interest in exploring the thermal properties of these frameworks, despite their relevance for practical adoption of MOFs as adsorbents. It is known that MOFs have a moderate thermal stability, ${ }^{11,12}$ a small heat capacity range ${ }^{11,13}$ and are considered to be poor conductors of heat, ${ }^{13,14}$ but most studies on thermal characteristics focus on a narrow set of well-known materials. The property that has received most attention is their thermal expansion behavior (e.g. ref. 15-18). Many frameworks display negative thermal expansion (NTE), i.e. the volume decreases when the temperature is increased..$^{13,19}$ While this anomalous behavior could be beneficial for the development of zero-expansion composites for highly-specialized applications, ${ }^{20}$ it poses some additional design constraints when in contact with conventional materials. Various practical ways of controlling the thermal expansion behavior of MOFs have already experimentally been demonstrated: retrofitting, ${ }^{21}$ adsorption of guest molecules, ${ }^{17,22,23}$ changing the organic linkers or metal ions, ${ }^{17,24,25}$ adding functional groups, ${ }^{16}$ incorporating defects, ${ }^{26}$ etc. Computational studies corroborate these findings. ${ }^{15,18,27,28}$

Thermal stress due to thermal expansion mismatch has received even less attention, ${ }^{29}$ but it was mentioned in the context of MOF thin film applications..$^{30,31}$ However, it could also be imagined to play a role when MOFs are used in confined environments such as volume-limited storage tank applications for natural gas and hydrogen storage. How thermal stress will 
impact the durability of the MOF crystals has not yet been investigated. Of course, the tolerable change of thermal expansion mismatch will also depend on the mechanical properties of the framework. It is already known that MOFs are mechanically not very stable, and much research into this is currently being performed. ${ }^{32-34}$

As a first step towards a better understanding of thermal stress effects, we provide in this work an estimate of the hypothetical thermal stress in closed storage systems by investigating the pressure necessary to keep the MOF from expanding or contracting when the temperature changes (Fig. 1(a)). In other words, we assume that the magnitude of thermal expansion of the MOF is much larger than the relative volume change of its surroundings. In practice, this will also depend on the contact material, but microscopic insight into solid/solid interfaces with MOFs is still in its infancy, except a number of studies on MOF/polymer interfaces for mixed matrix membranes. ${ }^{35}$ Therefore, we focus on the intrinsic characteristics of a pure MOF crystal and start our investigation by computing the thermal expansion coefficient of a selection of 52 rigid MOFs containing $\mathrm{Zn}, \mathrm{Cu}, \mathrm{Al}$ or $\mathrm{Zr}$ inorganic building blocks using force field molecular dynamics (Fig. 1(b)). These MOFs were all experimentally reported to possess good methane storage properties, ${ }^{36}$ which is necessary for several envisioned application areas of MOFs as efficient adsorbent. ${ }^{37,38}$ Subsequently, we compute the thermal pressure coefficient $\gamma$ as the product of the thermal expansion coefficient $\alpha_{0}$ and the bulk modulus $\beta_{0}$ (Fig. 1(c)). This quantity has mainly been considered in the context of thermal effects on the equation of state of solids, ${ }^{39,40}$ and we introduce it here as an intuitive indicator of the magnitude of isotropic thermal stress. We compare the thermal pressure coefficient with other materials, and with reported amorphization pressures of MOFs to get an intuitive understanding of its magnitude. This comparison suggests that variations within the operating temperature range of MOFs are expected to result in relatively low thermal stress. Finally, our results enable to identify some trends in terms of general MOF characteristics such as porosity and density.
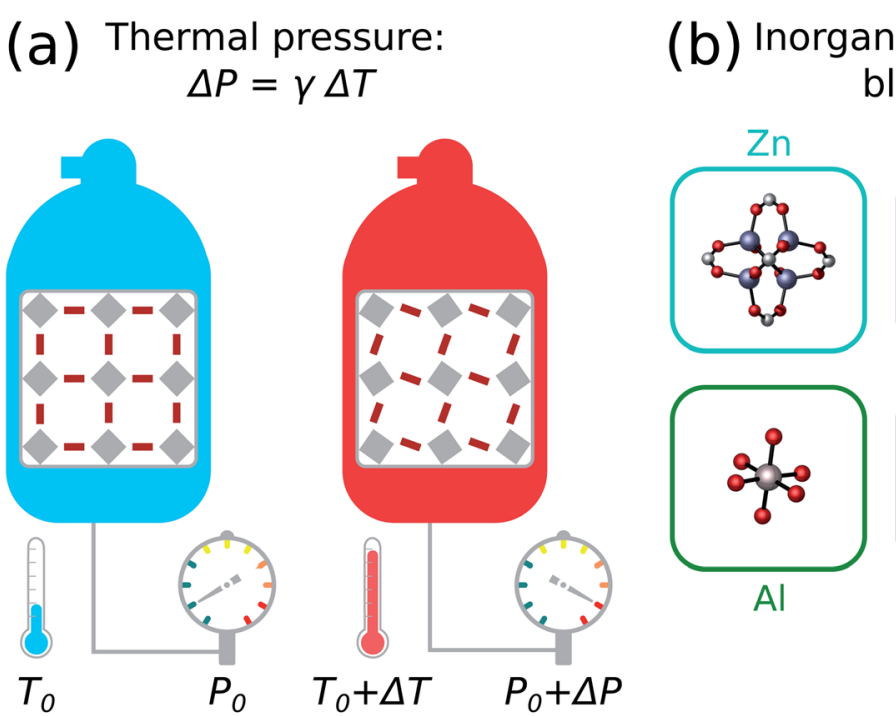

Al

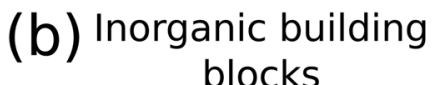
blocks

(C) Volume response $\Delta V$ upon external stimulus $(T, P)$

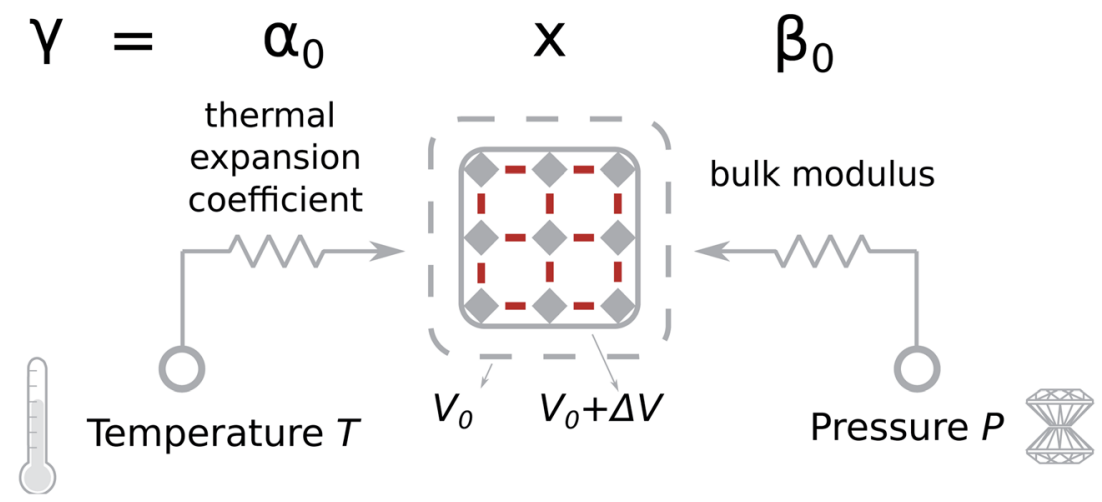

Fig. 1 (a) Thermal stress in a storage system subject to a temperature rise approximated with an isotropic thermal pressure. (b) MOFs containing zinc, copper, aluminum and zirconium inorganic building blocks were considered in this study. A full overview is given in Tables S1-S4 in the ESI. $\dagger$ (c) The thermal pressure coefficient $\gamma$ is an important parameter to quantify the isotropic thermal pressure, which is determined by the resistance to change volume upon temperature (thermal expansion coefficient $\alpha_{0}$ ) and pressure (bulk modulus $\beta_{0}$ ) stimuli. 


\section{Methods}

\subsection{Materials}

The 52 MOFs and their original references are listed in Tables S1-S4 in the ESI. $\dagger$

\subsection{Force field derivation}

The large unit cells of the MOFs under study make sufficiently long first-principles simulations impossible, and therefore we resorted to a more approximate technique, i.e. force fields. ${ }^{\mathbf{4 1}}$ The potential energy surface (PES) is then modelled by an analytical force field energy expression relying on a combination of covalent and noncovalent terms.

The covalent terms mimic the chemically bonded network, and the required covalent force field parameters were derived with our in-house developed QuickFF protocol. ${ }^{42,43}$ First, an accurate quantum mechanical PES is obtained using firstprinciples calculations on cluster models of the inorganic and organic building blocks of the MOFs. The required input data for the QuickFF protocol (equilibrium positions, and forces and Hessian calculated on this structure) are generated using the B3LYP exchange-correlation functional ${ }^{\mathbf{4 4}}$ as implemented in Gaussian $16 .^{45}$ Subsequently, using a fitting procedure, the unknown force constants are derived such that the force field PES approximates the quantum mechanical PES in the neighbourhood of the equilibrium structure. The force field expressions used for the approximation of the PES depend on the internal coordinates (bonds, bends, out-of-plane distances, dihedrals), and both anharmonic and cross terms are included in the force field. ${ }^{43}$

The noncovalent terms include the electrostatic and van der Waals interactions. Minimal Basis Iterative Stockholder (MBIS) charges are used to describe the Coulomb force between Gaussian charge distributions centered on the nuclei. ${ }^{46}$ These charges were derived from an all-electron density of the respective cluster model. Finally, the van der Waals interactions were modeled by the MM3-Buckingham $\operatorname{model}^{47}$ up to a finite cutoff of $12 \AA$ and were supplemented with tail corrections. ${ }^{48}$

\subsection{Molecular dynamics simulations}

Molecular dynamics (MD) simulations were performed in the isothermal-isobaric (NPT) ensemble. They were conducted at 1 bar and for temperatures in between $200 \mathrm{~K}$ and $400 \mathrm{~K}$ in steps of $50 \mathrm{~K}$.

The MD simulations were carried out with LAMMPS $^{49}$ using a Verlet time step of 0.5 fs. The temperature is controlled by a Nosé-Hoover chain thermostat ${ }^{50}$ containing three beads with a relaxation time of $0.1 \mathrm{ps}$, while the pressure is controlled by a Martyna-Tuckerman-Tobias-Klein barostat ${ }^{51}$ using a relaxation time of 1 ps. For most structures, $5 \mathrm{~ns}$ of data were obtained, while for the largest systems, 3 ns were used because of the larger computational cost. An equilibration time of $500 \mathrm{ps}$ was taken into account before extracting the properties from these MD simulations.

2.3.1 Thermal expansion coefficient. The thermal expansion coefficient $\alpha_{0}$ is defined as:

$$
\alpha_{0}=\frac{1}{V}\left(\frac{\partial V}{\partial T}\right)_{P} .
$$

We obtain $\alpha_{0}$ from the NPT MD simulations by fitting a linear polynomial to the ensemble averaged (indicated as $\langle\cdot\rangle$ ) natural logarithm of the volumes according to eqn (1):

$$
\langle\ln V\rangle \approx \alpha_{0} T+V_{0}
$$

where $V_{0}$ is the equilibrium volume at $0 \mathrm{~K}$. A comparison of computed thermal expansion coefficients with literature is provided in Table 1 . This suggests that we tend to predict slightly lower values for $\left|\alpha_{0}\right|$, which could be a result of neglecting nuclear quantum effects in our simulations. ${ }^{\mathbf{5 2 , 5 3}}$

The normal modes and corresponding frequencies to investigate the origin of the thermal expansion were derived using the harmonic approximation at $0 \mathrm{~K}$. The force field Hessian was computed with Yaff. ${ }^{54}$

2.3.2 Thermal pressure coefficient. The thermal pressure coefficient $\gamma$ is defined as:

$$
\gamma=\alpha_{0} \beta_{0}
$$

In other words, $\gamma$ is the product of the thermal expansion coefficient $\alpha_{0}$ and the bulk modulus $\beta_{0}$. To compute this quantity, we extracted the bulk modulus from the NPT MD simulations at $300 \mathrm{~K}$ using a fluctuation formula:

$$
\beta_{0}=k_{B} T \frac{\langle V\rangle}{\left\langle V^{2}\right\rangle-\langle V\rangle^{2}}
$$

where $k_{\mathrm{B}}$ is the Boltzmann constant. This relation was shown to provide a reliable result for MOFs, although more accurate estimations can be obtained at a substantially higher computational cost. ${ }^{73}$ A comparison of computed bulk moduli with literature is provided in Table 1 and shows that our results are in line with other reported values.

\subsection{Structural properties}

The structural properties (density $\rho$, accessible surface area (ASA), probe-occupiable void fraction, and largest cavity diameter (LCD)) were derived from the optimal structure at $0 \mathrm{~K}$ using Zeo++ ${ }^{76}\left(\mathrm{~N}_{2}\right.$ probe molecule with kinetic diameter $\left.3.64 \AA\right)$.

\section{Results}

\subsection{Thermal expansion}

Experimentally, thermal expansion behavior is typically studied using variable-temperature diffraction measurements. ${ }^{17,55,66}$ By collecting patterns at different temperatures, it becomes possible to fit the equilibrium volume as a function of temperature. In this work, we have used computational methods to extract the same information. Molecular dynamics simulations were employed in the isothermal-isobaric (NPT) ensemble. We determined the equilibrium volume $V$ at a mechanical pressure $P$ of 1 bar and for temperatures $T$ in a range from $200 \mathrm{~K}$ to $400 \mathrm{~K}$ in steps of $50 \mathrm{~K}$, and fitted them to 
Table 1 A comparison between thermal expansion coefficients $\alpha_{0}$ and bulk moduli $\beta_{0}$ computed in this work and reported in literature

\begin{tabular}{|c|c|c|c|c|c|c|}
\hline & $\alpha_{0}\left(\mathrm{MK}^{-1}\right)$ & $\alpha_{0}\left(\mathrm{MK}^{-1}\right)$ & $\alpha_{0}\left(\mathrm{MK}^{-1}\right)$ & $\begin{array}{l}\beta_{0} \\
(\mathrm{GPa})\end{array}$ & $\beta_{0}(\mathrm{GPa})$ & $\beta_{0}(\mathrm{GPa})$ \\
\hline MOF & This work & Experiment & Simulations & This work & Experiment & Simulations \\
\hline Al-soc-MOF-1 & -13 & - & $-19^{13}$ & 9 & - & - \\
\hline HKUST-1 & -10 & {$[-15,-12]^{21,55,56}$} & {$[-36,-9]^{13,41,57-59}$} & 21 & $30^{60}$ & {$[25-35]^{57-59,61}$} \\
\hline IRMOF-8 & -40 & - & {$[-32,-21]^{15,62}$} & 5 & - & {$[2,11]^{15,62,63}$} \\
\hline IRMOF-10 & -48 & - & {$[-55,-16]^{13,41,59,62}$} & 5 & - & {$[3,9]^{59,62-64}$} \\
\hline MOF-5 & -37 & {$[-48,-39]^{65,66}$} & {$[-79,-9]^{13,15,27,41,52,59,62,67,68}$} & 11 & - & {$[4,22]^{15,41,59,62-64,67,69-75}$} \\
\hline MOF-177 & -30 & - & {$[-39,-28]^{13,62}$} & 6 & - & $10^{74}$ \\
\hline MOF-205 & -29 & - & $-33^{18}$ & 7 & - & $11^{74}$ \\
\hline MOF-210 & -97 & - & $-55^{18}$ & 2 & - & - \\
\hline MOF-505 & -11 & - & $-17^{13}$ & 20 & - & - \\
\hline PCN-68 & -42 & - & $-54^{18}$ & 7 & - & - \\
\hline UMCM-1 & -41 & - & $-47^{13}$ & 6 & - & - \\
\hline
\end{tabular}

determine the well-known thermal expansion coefficient $\alpha_{0}$ (see eqn (1)).

This procedure was applied to a set of diverse MOFs known for their methane storage properties. ${ }^{36}$ All 52 materials are listed in Tables S1-S4 in the ESI. $\dagger$ To the best of our knowledge, this is the most comprehensive set to date for the topic under study. It does not cover the whole class of MOFs as it is merely a selection from experimentally reported structures, but contains different metal nodes $\left(\mathrm{Zn}_{4} \mathrm{O}, \mathrm{Cu}_{2} \mathrm{COO}_{4}, \mathrm{Zr}, \mathrm{Al}\right)($ Fig. 1(b)) and topological families.

A positive correlation between the magnitude of the thermal expansion coefficient and the pore size has already been demonstrated in other studies focusing on a particular topology ${ }^{15,27}$ or on a limited set of frameworks. ${ }^{17,18}$ Not surprisingly, we find for our materials a similar result in Fig. 2 where the absolute value of the thermal expansion coefficient as a function of the void fraction is displayed. Consistent with our

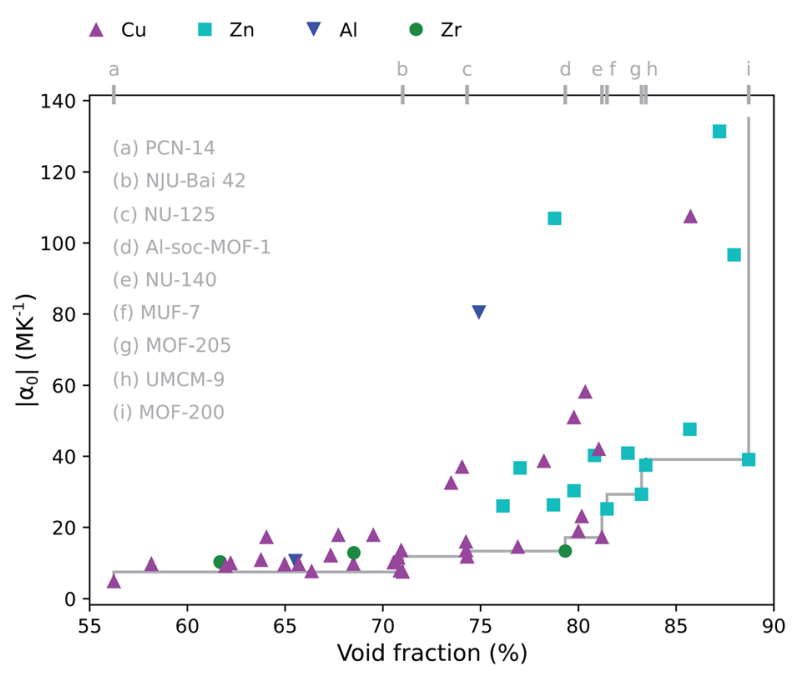

Fig. 2 The magnitude of the thermal expansion coefficient as a function of the void fraction. A Pareto front where the thermal expansion coefficient is minimized and the void fraction is maximised is shown. more diverse set of MOFs, a larger spread is observed, but the general trend remains preserved. Analogous relations are shown for the largest cavity diameter (Fig. S1 $\dagger$ ) and the accessible surface area (Fig. S2 $\dagger$ ) in the ESI. $\dagger$ Although Fig. 2 and S3 $\dagger$ seem to suggest that MOFs with a $\mathrm{Zn}_{4} \mathrm{O}$ inorganic building block tend to have a larger thermal expansion, this picture might be misleading as the investigated materials certainly do not cover the whole chemical space in terms of e.g. the void fraction. ${ }^{77}$ To investigate the effect of the chemical building blocks on the thermal expansion, the other building blocks and topology should ideally be kept fixed. ${ }^{17}$

All 52 frameworks in our set display NTE behavior in the temperature range from $200 \mathrm{~K}$ to $400 \mathrm{~K}$. We find thermal expansion coefficients in a wide range from $-5 \mathrm{MK}^{-1}$ to -131 $\mathrm{MK}^{-1}$ with an average of $-30 \mathrm{MK}^{-1}$ in Table 2 . The individual thermal expansion coefficients are tabulated for every MOF in Tables S1-S4 in the ESI. $\dagger$ These results again corroborate the widespread existence of NTE in network materials. ${ }^{19}$ Furthermore, we find three MOFs (MOF-180, ${ }^{78}$ MOF- $905{ }^{79}$ and PCN610/NU-100 ${ }^{80,81}$ ) exhibiting so-called colossal NTE behavior

Table 2 Summary of the properties of the MOFs under study. The structural properties (density $\rho$, accessible surface area (ASA), probeoccupiable void fraction, and largest cavity diameter (LCD)) were derived from the optimal structure at $0 \mathrm{~K}$ (see Methods section). The other properties are determined using molecular dynamics simulations at room temperature $(300 \mathrm{~K})$ : thermal expansion coefficient $\alpha_{0}$, bulk modulus $\beta_{0}$ and thermal pressure coefficient $\gamma$. The average $\left(\mu_{X}\right)$, the median $\left(m_{X}\right)$, the standard deviation $\left(\sigma_{X}\right)$ and the range $\left(\min _{X}\right.$ and $\left.\max _{x}\right)$ are tabulated. The results for every MOF are listed in Tables S1S4 in the ESI. A comparison of simulated $\alpha_{0}$ and $\beta_{0}$ with experiments is given in the ESI

\begin{tabular}{llllll}
\hline$X$ & $\mu_{X}$ & $m_{X}$ & $\sigma_{X}$ & $\min _{X}$ & $\max _{X}$ \\
\hline$\rho\left(\mathrm{kg} \mathrm{m}^{-3}\right)$ & 551 & 553 & 182 & 204 & 894 \\
ASA $\left(\mathrm{m}^{2} \mathrm{~g}^{-1}\right)$ & 3934 & 3970 & 1129 & 1163 & 6358 \\
Void fraction (\%) & 73 & 74 & 10 & 32 & 89 \\
LCD (̊) & 16 & 15 & 6 & 9 & 32 \\
$\alpha_{0}\left(\mathrm{MK}^{-1}\right)$ & -30 & -17 & 28 & -131 & -5 \\
$\beta_{0}(\mathrm{GPa})$ & 9 & 8 & 5 & 1 & 21 \\
$\left.\gamma(\mathrm{MPa} \mathrm{K})^{-1}\right)$ & -0.18 & -0.16 & 0.09 & -0.42 & -0.03
\end{tabular}


$\left(\left|\alpha_{0}\right|>100 \mathrm{MK}^{-1}\right)^{82}$ that might be worth exploring experimentally for specific applications. On the one hand, MOF-180 and PCN-610 contain among the largest tritopic and hexatopic organic linkers in our set, respectively, while MOF-905 has relatively short ditopic and tritopic organic linkers (Fig. S4 and Tables S6, S8†). On the other hand, other MOFs with a similar large linker length possess a relatively low coefficient $\left(\mathrm{MOF}-200^{78}\right)$. This suggests that having a very long organic linker is neither a sufficient nor a necessary condition for large thermal expansion. Furthermore, there are MOFs in our set with higher largest cavity diameters and void fractions, but still have much lower thermal expansion coefficients. It only illustrates the difficult relationship between the extent of negative thermal expansion and factors such as the length and type of the organic linker, and the topology.

Generally speaking, the magnitude of thermal expansion of MOFs is larger than most typical materials because of their large free voids. ${ }^{13}$ This does not pose a problem as long as the crystal can freely expand or contract in every direction. However, depending on the type of application, the material will be restricted to adapt in one or more directions with lower expansion coefficients as MOFs will be integrated in miniaturised devices connected to other materials and components. ${ }^{30,83-86}$ This restriction imposes thermal stress due to the thermal expansion mismatch. Only a limited number of experimental studies have actually monitored thermal expansion mismatch in MOFs. For instance, Wöll and co-workers demonstrated that a HKUST-1 thin film was stable under repeated heating-cooling cycles although the thermal expansion behavior of the MOF layer and substrate differ strongly. ${ }^{29}$ The same was observed in a different setup by the group of Weckhuysen. ${ }^{87}$

From a practical point of view, if several candidate MOFs perform similarly in terms of e.g. gas uptake, it will in many cases be beneficial to select the one with the lowest thermal expansion coefficient. One way of doing this is by constructing the Pareto front within a set of materials to optimize multiple objectives. ${ }^{\mathbf{8 8} 89}$ This is demonstrated for the thermal expansion and empty pore space in Fig. 2. It is not possible to find a MOF within the group of materials under study that has a lower thermal expansion and a higher void fraction than the MOFs on the Pareto front, which are listed in Fig. 2. For instance, this shows that it is possible to have a crystal with almost $90 \%$ empty space, while still having a moderate thermal expansion coefficient of $-40 \mathrm{MK}^{-1}$ (MOF-200).

The origin of the large NTE of MOFs has been ascribed to low-energy transverse motions. ${ }^{\mathbf{1 9 , 9 0 , 9 1}}$ To establish the link between the presence of collective motions with low frequencies and the relative thermal expansion, we computed the normal mode spectrum with the harmonic approximation. Fig. 3 illustrates that the relative number of low frequency modes is coupled with the thermal expansion behaviour, as MOFs displaying the largest thermal expansion coefficients possess the highest degree of low frequencies in their spectrum and vice versa. The indicated quadrants serve as a guide to the eye for this qualitative trend. The underlying mechanisms for NTE are framework dependent, and to elucidate them in-depth case

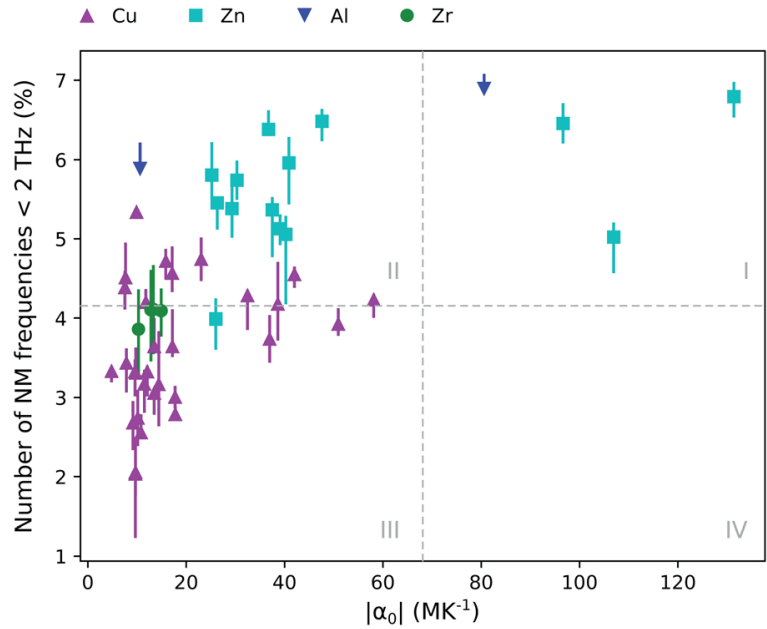

Fig. 3 Number of normal mode (NM) frequencies below $2 \mathrm{THz}$ (in \%) versus the magnitude of the thermal expansion coefficient $\left|\alpha_{0}\right|$. As these low vibrational modes can be prone to numerical noise, we multiplied all normal mode frequencies with 0.95 and 1.05, and computed again the number of frequencies below $2 \mathrm{THz}$ to estimate an error bar. The indicated quadrants serve as a guide to the eye.

studies are necessary as previously was demonstrated for MOF$5,{ }^{65,66}$ HKUST-1 ${ }^{55}$ and MIL-53(Al), ${ }^{91}$ for example. Further methodological developments in (computational) spectroscopy are required to systematically unravel the character of these important low-frequency vibrations. ${ }^{\mathbf{9 2 , 9 3}}$

In summary, our diverse set of MOFs again shows that the magnitude of thermal expansion is positively correlated with the porosity of the framework. The same holds to some extent for the presence of low vibrational modes. In other words, these findings thus indicate that in order to limit the thermal expansion mismatch with other materials (possessing much lower expansion coefficients), one should prefer the densest MOF structure in case various candidates display similar adsorption characteristics.

\subsection{Thermal pressure}

Thermal stress is intimately linked with the mechanical rigidity and stability of the material. ${ }^{\mathbf{9}}$ As many MOFs are prone to mechanical failure and a reduction in porosity during postsynthetic processing steps, it is essential to develop a good understanding of their mechanical properties. ${ }^{12,32,33}$ Thermal stress is a directional dependent quantity that can be calculated by multiplying the thermal expansion coefficient, the Young modulus and the temperature change. As MOFs can behave very anisotropically, ${ }^{95}$ we focus instead on the coefficient of the isotropic thermal pressure in this work (see eqn (3)).

The thermal pressure coefficient $\gamma$ provides information on the mechanical pressure rise in a closed, restricted system subjected to a uniform temperature rise as $\Delta P=\gamma \Delta T$. The product in eqn (3) combines the engineering parameters that describe how the volume of the material responds to pressure with its equivalent in terms of the temperature (Fig. 1(c)). Both 


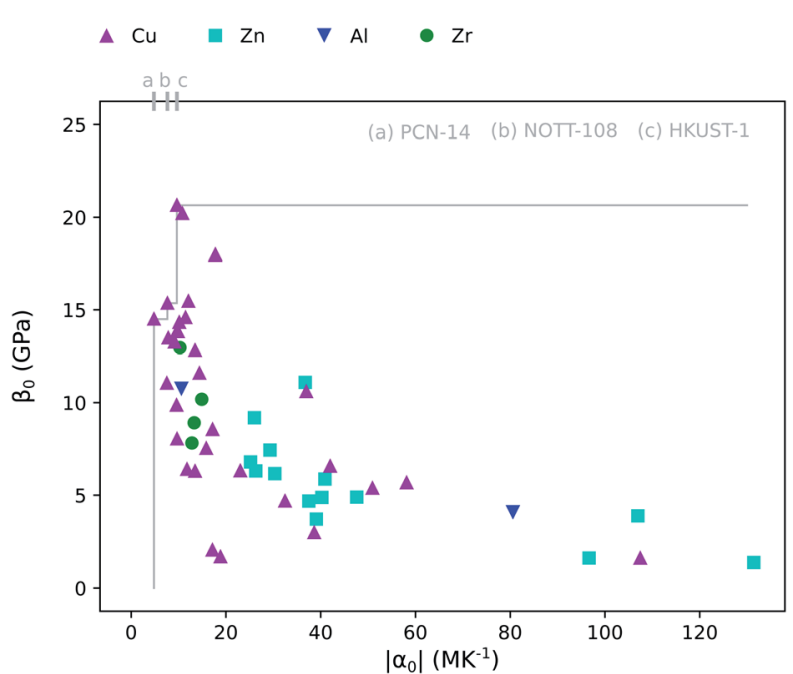

Fig. 4 Bulk modulus $\beta_{0}$ versus the magnitude of the thermal expansion coefficient $\left|\alpha_{0}\right|$. A Pareto front where $\left|\alpha_{0}\right|$ is minimized and $\beta_{0}$ is maximised is shown.

properties are of primary importance when considering devices where MOFs will come in contact with other materials.

They are not independent of each other. While the degree of thermal expansion is positively correlated with increasing porosity, the opposite is true for the bulk modulus. The latter was clearly illustrated by a large-scale computational screening of MOFs by Moghadam et al. ${ }^{34}$ As a result, the bulk modulus and the thermal expansion coefficient are roughly inversely correlated, which is obvious from the results shown in Fig. 4. Here, the bulk modulus is plotted versus the magnitude of the thermal expansion coefficient. On the one hand, relatively high bulk moduli of approximately $20 \mathrm{GPa}$ are found for copper-paddle wheel MOFs with limited NTE coefficients such as MOF-505/ NOTT- $100^{96,97}$ and HKUST- $1 .{ }^{98}$ For the latter material, experiment estimates a value of about $30 \mathrm{GPa} .{ }^{60}$ On the other hand, MOFs that display colossal NTE all have bulk moduli below $5 \mathrm{GPa}$.

The inverse relation between both $\alpha_{0}$ and $\beta_{0}$ results in a thermal pressure coefficient $\gamma$ that shows no correlation with typical framework characteristics such as void fraction, density or internal surface area. This is illustrated in Fig. 5, where $\gamma$ is plotted against the density. On average $\gamma$ is found to be $-0.18 \mathrm{MPa} \mathrm{K}^{-1}$ (Table 2), which corresponds with pulling the framework isotropically when the temperature increases. Of course, the minus sign - and thus tension instead of compression - comes from the NTE behavior.

The thermal pressure rise can finally be found by multiplying $\gamma$ with a temperature change $\Delta T$ (Fig. 1(a)). Of course, this depends on the application involving MOFs, and is only a rough estimate as both the bulk modulus and the thermal expansion coefficient are also influenced by guests and temperature. ${ }^{99}$ For example, consider the case of methane adsorption at room temperature. While only a few experimental studies have reported dynamical temperature changes due to the heat of adsorption, ${ }^{7,8}$ it can be expected that even at very high gas

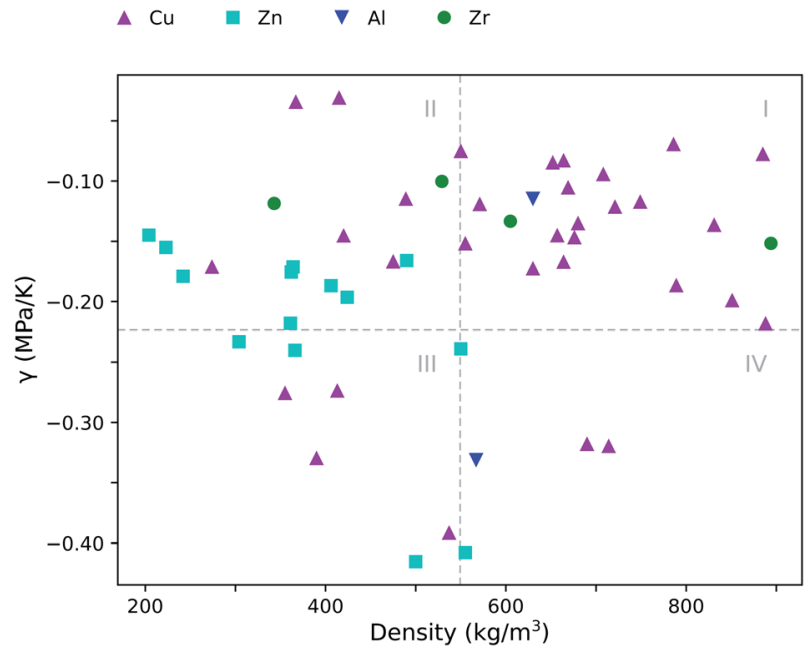

Fig. 5 Thermal pressure coefficient $\gamma$ as a function of the density $\rho$. The indicated quadrants serve as a guide to the eye.

pressures this will not go beyond $100 \mathrm{~K} \cdot \cdot^{13}$ For a hypothetical setup with MOFs confined and restricted in a closed system, this would maximally result in a thermal pressure rise between $-42 \mathrm{MPa}$ and $-5 \mathrm{MPa}$ for the materials under study (Table 2). This is relatively small compared to amorphization pressures that lead to pore collapse during compression (from $50 \mathrm{MPa}$ to pressures beyond $1 \mathrm{GPa}) \cdot{ }^{34,100}$

\section{Discussion}

Finally, we compare our simulated data with other conventional materials in Fig. 6 to better understand the magnitude of the thermal pressure coefficients. Mechanical and thermal properties of MOFs have been situated at the border of polymers and ceramics in the past. ${ }^{13,32}$ The same can now be said for $\gamma$, which is a quantity combining both thermal and mechanical properties. On the one hand, polymers tend to have higher thermal expansion coefficients, but they are also mechanically weaker. Ceramics, on the other hand, have typically lower thermal expansion coefficients than MOFs, but have a wide range of bulk moduli. It is clear, however, that the thermal pressure coefficients of metals are an order of magnitude larger.

From Fig. 6, it is obvious that there is a link between the thermal pressure coefficient and the density of a material on a larger scale. The simulated results for the MOFs under study fit in this trend. This relation can intuitively be understood as $\gamma$ can also be written using the Maxwell relations as:

$$
\gamma=\left(\frac{\partial S}{\partial V}\right)_{T} .
$$

A dense material will typically require a smaller volume change to instigate the same entropy change as a less dense material. 


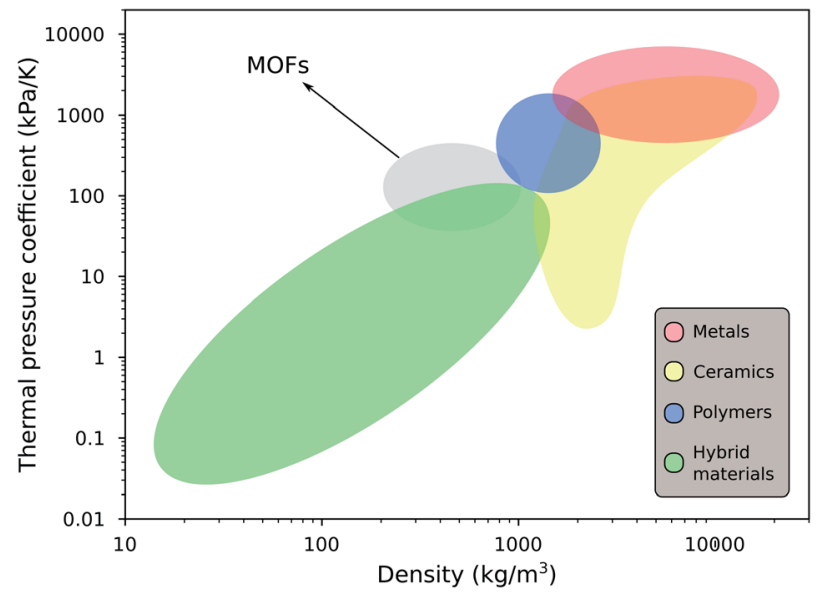

Fig. 6 Materials property chart displaying the magnitude of the thermal pressure coefficient $\gamma$ as a function of the density of MOFs alongside other material classes, namely, metals (and alloys), ceramics (glasses and (non-)technical ceramics), polymers and hybrid materials (composites, foams and natural materials).

\section{Conclusions}

In summary, this work investigated the thermal expansion behavior and its link with thermal stress. On a set consisting of more than fifty MOFs reported for methane storage, we demonstrate that the magnitude of the thermal expansion coefficient is positively correlated with the porosity of the framework. We predict for all materials NTE with three frameworks displaying a colossal volume change around room temperature. Although in-depth studies will be required to understand the link between specific collective motions and thermal expansion, we show that a relatively high percentage of low vibrational modes in the normal mode spectrum is an indicator for potentially large temperature-induced volume changes.

As a first measure for thermal stress, we introduce the thermal pressure coefficient $\gamma$, which is the product of the thermal expansion coefficient and the bulk modulus. As both properties are roughly inversely correlated, we do not find a clear link between framework characteristics and $\gamma$. The simulated thermal pressure coefficients $\gamma$ of the investigated MOFs were found to be relatively low, in between the range of polymers and ceramics. Systems working near thermal equilibrium conditions where MOF crystals are restricted in volume change (e.g. due to thermal expansion mismatch) are predicted to produce thermal pressure rises of only a couple of megapascals. The materials are then subjected to an isotropic pulling force much lower than typical amorphization pressures, and are therefore not expected to easily fail.

\section{Conflicts of interest}

There are no conflicts to declare.

\section{Acknowledgements}

This work is supported by the Fund for Scientific Research Flanders (FWO). V. V. S. acknowledges funding from the
European Union's Horizon 2020 research and innovation program (consolidator ERC grant agreement no. 647755 DYNPOR (2015-2020)). The work is furthermore supported by the Research Board of Ghent University (BOF). The computational resources and services used in this work were provided by VSC (Flemish Supercomputer Center), funded by Ghent University, FWO and the Flemish Government department EWI.

\section{Notes and references}

1 K. Chen, J. Cole, C. Conger, J. Draskovic, M. Lohr, K. Klein, T. Scheidemantel and P. Schiffer, Nature, 2006, 442, 257.

2 G. Abadias, J. Vac. Sci. Technol., 2018, 36, 020801.

3 J. D. Steele, J. Handong, I. Dovgaliuk, R. F. Berger, T. Braeckevelt, H. Yuan, C. Martin, E. Solano, K. Lejaeghere, S. M. J. Rogge, C. Notebaert, W. Vandezande, K. P. F. Janssen, B. Goderis, E. Debroye, Y.-K. Wang, Y. Dong, D. Ma, M. Saidaminov, H. Tan, Z. Lu, V. Dyadkin, D. Chernyshov, V. Van Speybroeck, E. H. Sargent, J. Hofkens and M. B. Roeffaers, Science, 2019, 365, 679-684.

4 H. Furukawa, K. E. Cordova, M. O'Keeffe and O. M. Yaghi, Science, 2013, 341, 1230444.

5 P. Silva, S. M. F. Vilela and F. A. Almeida Paz, Chem. Soc. Rev., 2015, 44, 6774-6803.

6 C. A. Trickett, A. Helal, B. A. Al-Maythalony, Z. H. Zamani, K. E. Cordova and O. M. Yaghi, Nat. Rev. Mater., 2017, 2, 17045.

7 V. Bon, N. Kavoosi, I. Senkovska and S. Kaskel, ACS Appl. Mater. Interfaces, 2015, 7, 222292-222300.

8 M. Beckner and A. Dailly, Appl. Energy, 2016, 162, 506-514. 9 J. Xiao, M. Hu, P. Bénard and R. Chahine, Int. J. Hydrogen Energy, 2013, 38, 13000-13010.

10 J. A. Mason, K. Sumida, Z. R. Herm, R. Krishna and J. R. Long, Chem. Sci., 2011, 4, 3030-3040.

11 B. $\mathrm{Mu}$ and K. S. Walton, J. Phys. Chem. C, 2011, 115, 2274822754.

12 A. J. Howarth, Y. Liu, P. Li, Z. Li, T. C. Wang, J. T. Hupp and O. K. Farha, Nat. Rev. Mater., 2016, 1, 15018.

13 J. Wieme, S. Vandenbrande, A. Lamaire, V. Kapil, L. Vanduyfhuys and V. Van Speybroeck, ACS Appl. Mater. Interfaces, 2019, 11, 38697-38707.

14 H. Babaei, A. J. H. McGaughey and C. E. Wilmer, Chem. Sci., 2017, 8, 583-589.

15 S. S. Han and W. A. Goddard, J. Phys. Chem. C, 2007, 111, 15185-15191.

16 S. Henke, A. Schneemann and R. A. Fischer, Adv. Funct. Mater., 2013, 23, 5990-5996.

17 N. C. Burtch, S. J. Baxter, J. Heinen, A. Bird, A. Schneemann, D. Dubbeldam and A. P. Wilkinson, Adv. Funct. Mater., 2019, 1904669.

18 J. D. Evans, J. P. Dürholt, S. Kaskel and R. Schmid, J. Mater. Chem. A, 2019, 7, 24019-24026.

19 C. S. Coates and A. L. Goodwin, Mater. Horiz., 2019, 6, 211218.

20 G. D. Barrera, J. A. O. Bruno, T. H. K. Barron and N. L. Allan, J. Phys.: Condens. Matter, 2005, 17, 4. 
21 C. Schneider, D. Bodesheim, M. G. Ehrenreich, V. Crocellà, J. Mink, R. A. Fischer, K. T. Butler and G. Kieslich, J. Am. Chem. Soc., 2019, 141, 10504.

22 I. Grobler, V. J. Smith, P. M. Bhatt, S. A. Herbert and L. J. Barbour, J. Am. Chem. Soc., 2013, 135, 6411-6414.

23 J. E. Auckett, A. A. Barkhordarian, S. H. Ogilvie, S. G. Duyker, H. Chevreau, V. K. Peterson and C. J. Kepert, Nat. Commun., 2018, 9, 4873.

24 C. Nanthamathee, S. Ling, B. Slater and M. P. Attfield, Chem. Mater., 2015, 27, 85-95.

25 S. J. Baxter, A. Scheemann, A. D. Ready, P. Wijeratne, A. P. Wilkinson and N. C. Burtch, J. Am. Chem. Soc., 2019, 141, 12849-12854.

26 M. J. Cliffe, J. A. Hill, C. A. Murray, F.-X. Coudert and A. L. Goodwin, Phys. Chem. Chem. Phys., 2015, 17, 1158611592.

27 D. Dubbeldam, K. S. Walton, D. E. Ellis and R. Q. Snurr, Angew. Chem., Int. Ed., 2007, 46, 4496-4499.

28 S. R. G. Balestra, R. Bueno-Perez, S. Hamad, D. Dubbeldam, R. A. Ruiz-Salvador and S. Calero, Chem. Mater., 2016, 28, 8296-8304.

29 Z. Wang, P. G. Weidler, C. Azucena, L. Heinke and C. Wöll, Microporous Mesoporous Mater., 2016, 222, 241-246.

30 I. Stassen, N. Burtch, A. Talin, P. Falcaro, M. Allendorf and R. Ameloot, Chem. Soc. Rev., 2017, 46, 3185.

31 N. C. Burtch, J. Heinen, T. D. Bennett, D. Dubbeldam and M. D. Allendorf, Adv. Mater., 2018, 30, 1704124.

32 J. C. Tan and A. K. Cheetham, Chem. Soc. Rev., 2011, 40, 1059-1080.

33 L. R. Redfern and O. K. Farha, Chem. Sci., 2019, 10, 1066610679.

34 P. Z. Moghadam, S. M. J. Rogge, A. Li, C.-M. Chow, J. Wieme, N. Moharrami, M. Aragones-Anglada, G. Conduit, D. A. Gomez-Gualdron, V. Van Speybroeck and D. Fairen-Jimenez, Matter, 2019, 1, 219-234.

35 R. Semino, N. A. Ramsahye, A. Ghoufi and G. Maurin, J. Phys. Chem. C, 2016, 8, 809-819.

36 Y. He, F. Chen, B. Li, G. Qian, W. Zhou and B. Chen, Coord. Chem. Rev., 2017, 373, 167-198.

37 C. M. Simon, J. Kim, D. A. Gomez-Gualdron, J. S. Camp, Y. G. Chung, R. L. Martin, R. Mercado, M. W. Deem, D. Gunter, M. Haranczyk, D. S. Sholl, R. Q. Snurr and B. Smit, Energy Environ. Sci., 2015, 8, 1190-1199.

38 J. A. Mason, J. Oktawiec, M. K. Taylor, M. R. Hudson, J. Rodriguez, J. E. Bachman, M. I. Gonzalez, A. Cervellino, A. Guagliardi, C. M. Brown, P. L. Llewellyn, N. Masciocchi and J. R. Long, Nature, 2015, 527, 357-361.

39 O. L. Anderson, J. Phys. Chem. Solids, 1997, 58, 335-343.

40 T. J. B. Holland and R. Powell, J. Struct. Geol., 2011, 29, 333383.

41 P. G. Boyd, S. M. Moosavi, M. Witman and B. Smit, J. Phys. Chem. Lett., 2017, 8, 357-363.

42 L. Vanduyfhuys, S. Vandenbrande, T. Verstraelen, R. Schmid, M. Waroquier and V. Van Speybroeck, J. Comput. Chem., 2015, 36, 1015-1027.
43 L. Vanduyfhuys, S. Vandenbrande, J. Wieme, M. Waroquier, T. Verstraelen and V. Van Speybroeck, J. Comput. Chem., 2018, 39, 999-1011.

44 A. D. Becke, J. Chem. Phys., 1993, 98, 5648-5652.

45 M. J. Frisch, G. W. Trucks, H. B. Schlegel, G. E. Scuseria, M. A. Robb, J. R. Cheeseman, G. Scalmani, V. Barone, G. A. Petersson, H. Nakatsuji, X. Li, M. Caricato, A. V. Marenich, J. Bloino, B. G. Janesko, R. Gomperts, B. Mennucci, H. P. Hratchian, J. V. Ortiz, A. F. Izmaylov, J. L. Sonnenberg, D. Williams-Young, F. Ding, F. Lipparini, F. Egidi, J. Goings, B. Peng, A. Petrone, T. Henderson, D. Ranasinghe, V. G. Zakrzewski, J. Gao, N. Rega, G. Zheng, W. Liang, M. Hada, M. Ehara, K. Toyota, R. Fukuda, J. Hasegawa, M. Ishida, T. Nakajima, Y. Honda, O. Kitao, H. Nakai, T. Vreven, K. Throssell, J. A. Montgomery, Jr, J. E. Peralta, F. Ogliaro, M. J. Bearpark, J. J. Heyd, E. N. Brothers, K. N. Kudin, V. N. Staroverov, T. A. Keith, R. Kobayashi, J. Normand, K. Raghavachari, A. P. Rendell, J. C. Burant, S. S. Iyengar, J. Tomasi, M. Cossi, J. M. Millam, M. Klene, C. Adamo, R. Cammi, J. W. Ochterski, R. L. Martin, K. Morokuma, O. Farkas, J. B. Foresman and D. J. Fox, Gaussian 16 Revision E.01, Gaussian Inc., Wallingford CT, 2016.

46 T. Verstraelen, S. Vandenbrande, F. Heidar-Zadeh, L. Vanduyfhuys, V. Van Speybroeck, M. Waroquier and P. W. Ayers, J. Chem. Theory Comput., 2016, 12, 3894-3912. 47 J. H. Lii and N. L. Allinger, J. Am. Chem. Soc., 1989, 111, 8576-8582.

48 H. Sun, J. Phys. Chem. B, 1998, 102, 7338-7364.

49 S. Plimpton, J. Comput. Phys., 1995, 117, 1-19.

50 G. J. Martyna, M. L. Klein and M. Tuckerman, J. Chem. Phys., 1992, 97, 2635-2643.

51 G. J. Martyna, M. E. Tuckerman, D. J. Tobias and M. L. Klein, Mol. Phys., 1996, 87, 1117-1157.

52 A. Lamaire, J. Wieme, S. M. J. Rogge, M. Waroquier and V. Van Speybroeck, J. Chem. Phys., 2019, 150, 094503.

53 V. Kapil, J. Wieme, S. Vandenbrande, A. Lamaire, V. Van Speybroeck and M. Ceriotti, J. Chem. Theory Comput., 2019, 15, 3237-3249.

54 T. Verstraelen, L. Vanduyfhuys, S. Vandenbrande and S. M. J. Rogge, Yaff 1.5.0, 2019, http://molmod.github.io/ yaff/index.html.

55 Y. Wu, A. Kobayashi, G. J. Halder, V. K. Peterson, K. W. Chapman, N. Lock, P. D. Southon and C. J. Kepert, Angew. Chem., Int. Ed., 2008, 47, 8929-8932.

56 V. K. Peterson, G. J. Kearley, Y. Wu, A. J. Ramirez-Cuesta, E. Kemner and C. J. Kepert, Angew. Chem., Int. Ed., 2010, 49, 585-588.

57 L. Zhao, Q. Yang, Q. Ma, C. Zhong, J. Mi and D. Liu, J. Mol. Model., 2011, 17, 227-234.

58 M. Tafipolsky, S. Amirjalayer and R. Schmid, J. Phys. Chem. C, 2010, 114, 14402-14409.

59 J. K. Bristow, D. Tiana and A. Walsh, J. Chem. Theory Comput., 2014, 10, 4644-4652.

60 K. W. Chapman, G. J. Halder and P. J. Chupas, J. Am. Chem. Soc., 2008, 10524-10526. 
61 H. Wu, T. Yildirim and W. Zhou, J. Phys. Chem. Lett., 2013, 4, 925-930.

62 Y. Sun and H. Sun, J. Mol. Model., 2014, 20, 2146.

63 A. Kuc, A. Enyashin and G. Seifert, J. Phys. Chem. B, 2007, 111, 8179-8186.

64 K. Banlusan and A. Strachan, J. Chem. Phys., 2017, 146, 184705.

65 W. Zhou, H. Wu, T. Yildirim, J. R. Simpson and A. R. Hight Walker, Phys. Rev. B: Condens. Matter Mater. Phys., 2008, 78, 054114.

66 N. Lock, Y. Wu, M. Christensen, L. J. Cameron, V. K. Peterson, A. J. Bridgeman, C. J. Kepert and B. B. Iversen, J. Phys. Chem. C, 2010, 114, 16181-16186.

67 J. A. Greathouse and M. D. Allendorf, J. Phys. Chem. C, 2008, 112, 5795-5802.

68 M. R. Ryder, J. Maul, B. Civalleri and A. Erba, Adv. Theory Simul., 2019, 2, 1900093.

69 W. Zhou and T. Yildirim, Phys. Rev. B: Condens. Matter Mater. Phys., 2006, 74, 180301.

70 A. Samanta, T. Furuta and J. Li, J. Chem. Phys., 2006, 125, 084714.

71 M. Tafipolsky and R. Schmid, J. Phys. Chem. B, 2009, 113, 1341-1352.

72 D. F. Bahr, J. A. Reid, W. M. Mook, C. A. Bauer, R. Stumpf, A. J. Skulan, N. R. Moody, B. A. Simmons, M. M. Shindel and M. D. Allendorf, Phys. Rev. B: Condens. Matter Mater. Phys., 2008, 76, 059902.

73 S. M. J. Rogge, L. Vanduyfhuys, A. Ghysels, M. Waroquier, T. Verstraelen, G. Maurin and V. Van Speybroeck, J. Chem. Theory Comput., 2015, 11, 12.

74 B. Lukose, B. Supronowicz, P. S. Petkov, J. Frenzel, A. B. Kuc, G. Seifert, G. N. Vayssilov and T. Heine, Phys. Status Solidi B, 2012, 249, 335-342.

75 M. Mattesini, J. M. Soler and F. Ynduráin, Phys. Rev. B: Condens. Matter Mater. Phys., 2006, 73, 094111.

76 T. F. Willems, C. H. Rycroft, M. Kazi, J. C. Meza and M. Haranczyk, Microporous Mesoporous Mater., 2012, 149, 134-141.

77 S. M. Moosavi, A. Nandy, K. M. Jablonka, D. Ongari, J. P. Janet, P. G. Boyd, Y. Lee, B. Smit and H. J. Kulik, Nat. Commun., 2020, 11, 4068.

78 H. Furukawa, N. Ko, Y. B. Go, N. Aratani, S. B. Choi, E. Choi, O. A. Yazaydin, R. Q. Snurr, M. O'Keeffe, J. Kim and O. M. Yaghi, Science, 2010, 329, 424-428.

79 J. Jiang, H. Furukawa, Y.-B. Zhang and O. M. Yaghi, J. Am. Chem. Soc., 2016, 138, 10244-10251.
80 D. Yuan, D. Zhao, D. Sun and H.-C. Zhou, Angew. Chem., Int. Ed., 2010, 49, 5357-5361.

81 O. K. Farha, O. A. Yazaydin, I. Eryazici, C. D. Malliakas, B. G. Hauser, M. G. Kanatzidis, S. T. Nguyen, R. Q. Snurr and J. T. Hupp, Nat. Chem., 2010, 2, 944-948.

82 A. L. Goodwin, D. A. Keen, M. G. Tucker, M. T. Dove, L. Peters and J. S. O. Evans, J. Am. Chem. Soc., 2008, 130, 9660-9661.

83 A. Bétard and R. A. Fischer, Chem. Rev., 2012, 112, 10551083.

84 Q.-L. Zhu and Q. Xu, Chem. Soc. Rev., 2014, 43, 5468-5512. 85 P. Falcaro, R. Ricco, C. M. Doherty, K. Liang, A. J. Hill and M. J. Styles, Chem. Soc. Rev., 2014, 43, 5513-5560.

86 J. Liu and C. Wöll, Chem. Soc. Rev., 2017, 46, 5730-5770.

87 R. P. Brand, L. D. B. Mandemaker, G. Delen, N. Rijnveld and B. M. Weckhuysen, Chem.-Eur. J., 2018, 19, 2397-2404.

88 J. Greeley, T. F. Jaramillo, J. Bonde, I. Chorkendorff and J. Nørskov, Nat. Mater., 2006, 5, 909-913.

89 K. Lejaeghere, S. Cottenier and V. Van Speybroeck, Phys. Rev. Lett., 2013, 111, 075501.

90 M. R. Ryder, B. Van de Voorde, B. Civalleri, T. D. Bennett, S. Mukhopadhyay, G. Cinque, F. Fernandez-Alonso, D. De Vos, S. Rudić and J.-C. Tan, Phys. Rev. Lett., 2017, 118, 255502.

91 A. E. J. Hoffman, J. Wieme, S. M. J. Rogge, L. Vanduyfhuys and V. Van Speybroeck, Z. Kristallogr. - Cryst. Mater., 2019, 234, 529-545.

92 P. U. Jepsen and S. J. Clark, Chem. Phys. Lett., 2007, 442, 275-280.

93 C. R. Jacob and M. Reiher, J. Chem. Phys., 2009, 130, 084106.

94 A. M. Evans, M. R. Ryder, N. C. Flanders, E. Vitaku, L. X. Chen and W. R. Dichtel, Ind. Eng. Chem. Res., 2019, 58, 9883-9887.

95 A. U. Ortiz, A. Boutin, A. H. Fuchs and F.-X. Coudert, Phys. Rev. Lett., 2012, 109, 195502.

96 B. Chen, N. W. Ockwig, A. R. Millward, D. S. Contreras and O. M. Yaghi, Angew. Chem., Int. Ed., 2005, 44, 4745-4749.

97 X. Lin, J. Jia, X. Zhao, M. Thomas, A. J. Blake, G. S. Walker, N. R. Champness, P. Hubberstey and M. Schröder, Angew. Chem., Int. Ed., 2006, 45, 7358-7364.

98 S. S.-Y. Chui, S. M.-F. Lo, J. P. H. Charmant, G. A. Orpen and I. D. Williams, Science, 1999, 283, 1148-1150.

99 J. Heinen, A. D. Ready, T. D. Bennett, D. Dubbeldam, R. W. Friddle and N. C. Burtch, ACS Appl. Mater. Interfaces, 2018, 10, 21079-21083.

100 T. D. Bennett and A. K. Cheetham, Acc. Chem. Res., 2014, 47, 1555-1562. 